\title{
POLUIÇÃO DAS ÁGUAS POR HERBICIDAS UTILIZADOS NO CULTIVO DO ARROZ IRRIGADO NA REGIÃO CENTRAL DO ESTADO DO RIO GRANDE DO SUL, BRASIL: PREDIÇÃO TEÓRICA E MONITORAMENTO
}

\author{
Ednei Gilberto Primel* \\ Departamento de Química, Fundação Universidade Federal do Rio Grande, 96201-900 Rio Grande - RS \\ Renato Zanella, Márcia Helena Scherer Kurz e Fábio Ferreira Gonçalves \\ Departamento de Química, Universidade Federal de Santa Maria, 97105-900 Santa Maria - RS \\ Sérgio de Oliveira Machado e Enio Marchezan \\ Departamento de Fitotecnia, Universidade Federal de Santa Maria, 97105-900 Santa Maria - RS
}

Recebido em 29/4/04; aceito em 19/11/04; publicado na web em 13/4/05

\begin{abstract}
POLLUTION OF WATER BY HERBICIDES USED IN THE IRRIGATED RICE CULTIVATION IN THE CENTRAL AREA OF RIO GRANDE DO SUL STATE, BRAZIL: THEORETICAL PREDICTION AND MONITORING. This article presents an evaluation of the pollution of river water by herbicides used in the culture of irrigated rice in Rio Grande do Sul State, Brazil. Firstly, a theoretical evaluation was made using the approaches suggested by EPA-USA, the "Groundwater Ubiquity Score" index and the Goss method to estimate the pollution possibilities. Afterwards, a monitoring program was established for the rivers of the area from 2001 to 2003 to investigate the presence of herbicide residues. The results indicate that the herbicides clomazone and propanil are the ones with larger presence and frequency in the analyzed samples. The theoretical forecast was confirmed by the results of the monitoring program.
\end{abstract}

Keywords: herbicides; water pollution; irrigated rice fields.

\section{INTRODUÇÃO}

Desde o início de seu desenvolvimento, a produção agrícola está diretamente relacionada com a aplicação de pesticidas para controlar as pestes que atacam os produtos agrícolas, prejudicando as colheitas. A aplicação de pesticidas gera, comumente, grandes problemas: os pesticidas muitas vezes são tóxicos, podendo ser cancerígenos, mutagênicos, teratogênicos e mimetizadores de hormônios ${ }^{1}$; são aplicados em grande quantidade, em áreas bastante extensas e, geralmente, possuem grande persistência no meio ambiente $^{2}$, além de gerar sérios problemas de qualidade das águas superficiais e subterrâneas ${ }^{3}$. O efeito e a magnitude decorrentes do uso de pesticidas no ambiente dependem basicamente dos processos de transferência e transformações que ocorrem em cada compartimento do sistema solo-água-planta-atmosfera ${ }^{4,5}$.

No cultivo de qualquer sistema agrícola, como por ex., no arroz irrigado em sistema de cultivo pré-germinado, o ponto de partida para o desencadeamento de impacto ambiental é a técnica de aplicação do pesticida. As maiores rotas de dispersão de pesticidas para sistemas aquáticos são o escoamento superficial e a drenagem. O tipo de planta e a topografia do terreno têm importância decisiva na maioria desses processos ${ }^{6}$. Assim, a dinâmica dos pesticidas no solo está relacionada com a precipitação pluvial e o manejo da irrigação ${ }^{7}$.

No sistema pré-germinado, a drenagem da área irrigada, efetuada após a semeadura, pode desencadear grave problema ambiental, ao mesmo tempo que pode causar perdas dos nutrientes e/ou de herbicidas que estão em suspensão na água de irrigação que é liberada. Com o decorrer do tempo, as vantagens do sistema são suplantadas pelas desvantagens ambientais.

*e-mail: dqmednei@ furg.br
Aproximadamente um terço de todos os compostos orgânicos produzidos têm como destino o meio ambiente, incluindo a água. Cerca de 700 compostos químicos, incluindo mais de 600 compostos orgânicos, muitos dos quais biologicamente ativos, têm sido detectados em amostras de água ${ }^{2}$.

O Brasil, desde a década de 70, destaca-se como um dos maiores consumidores mundiais de pesticidas ${ }^{8}$. Porém, o único dado que nos dá uma indicação da escala em que são aplicados no Brasil são os valores de pesticidas em linha de comercialização, a partir dos quais destaca-se a grande utilização de herbicidas ${ }^{9}$. As culturas responsáveis por este elevado consumo são principalmente soja, cana-de-açúcar, milho e arroz ${ }^{10}$. O estado do Rio Grande do Sul (RS) é responsável pela utilização de cerca de $20 \%$ dos pesticidas consumidos no país.

No Brasil são cultivados anualmente 1,3 milhões de hectares com arroz irrigado, dos quais cerca de 950 mil (73\%) estão no $\mathrm{RS}^{11}$. A orizicultura gaúcha contribui com cerca de $50 \%$ da produção nacional de arroz ${ }^{12}$. Apesar da grande contribuição do estado do RS na produção de arroz, muito pouco se fez até o momento em relação a estudos de comportamento e destino dos herbicidas no sistema, visando a manutenção da eficiência com menor risco de dano ambiental ${ }^{5}$.

Esse trabalho tem por objetivo avaliar o potencial teórico de lixiviação e o risco de poluição das águas por herbicidas utilizados no cultivo do arroz irrigado na região central do RS, usando para esta avaliação os critérios de "screening" da US-EPA; o índice GUS de vulnerabilidade de águas subterrâneas ("Groundwater Ubiquity Score, GUS"); e o método de Goss. Todos esses procedimentos levam em consideração as propriedades físico-químicas dos herbicidas e as propriedades do solo. Objetivou-se ainda comparar esta avaliação teórica com os resultados obtidos das análises de amostras de águas de rios, coletadas na região em estudo durante o período de cultivo do arroz irrigado nas safras agrícolas de 2000/ 01, 2001/02 e 2002/03. 


\section{PARTE EXPERIMENTAL}

\section{Área do estudo}

O estudo foi realizado a campo em área de várzea do Departamento de Fitotecnia da Universidade Federal de Santa Maria (UFSM), em Santa Maria (RS); e na Bacia Hidrográfica dos Rios Vacacaí e Vacacaí-Mirim durante o período de cultivo do arroz irrigado nas safras agrícolas de 2000/01, 2001/02 e 2002/03.

As coordenadas geográficas da região utilizada para o estudo são latitude $29^{\circ} 41^{\prime} 24^{\prime \prime} \mathrm{S}$, longitude $53^{\circ} 48^{\prime} 42^{\prime}$ 'W e altitude média de $95 \mathrm{~m}$. O solo é classificado como Planossolo Hidromórfico Eutrófico Arênico, pertencente à unidade de mapeamento Vacacaí. Apresenta relevo plano a suavemente ondulado, substrato de sedimentos aluviais recentes. No sistema americano é enquadrado como Albaqualf. Em geral, essa unidade de mapeamento apresenta textura média (15 a 25\% de argila), com baixos a médios teores de matéria orgânica, pobres em nutrientes e mal drenados, bastante influenciados pela presença de água, condicionada pelo relevo.

O clima é classificado como subtropical úmido, classe ' $\mathrm{Cfa}$ '. A precipitação pluvial média anual normal varia de 1.322 a 1.769 mm. A temperatura varia em média de $14,1^{\circ} \mathrm{C}$ no mês de julho a $24,9{ }^{\circ} \mathrm{C}$ em janeiro. Os valores extremos das médias das temperaturas máximas e mínimas no mês de julho são de 19,8 e $9,3{ }^{\circ} \mathrm{C}$, e em janeiro, 31,5 e $18,8{ }^{\circ} \mathrm{C}$, respectivamente.

A radiação média mensal oscila de 199 em julho a $518 \mathrm{cal} \mathrm{cm}^{-2}$ $\mathrm{dia}^{-1}$ em dezembro. A umidade relativa do ar varia de 83,8 em julho a $72,5 \%$ em dezembro e a precipitação pluvial varia de $111 \mathrm{em}$ novembro a $177 \mathrm{~mm}$ em outubro. Os valores máximos de evapotranspiração potencial são da ordem de $138 \mathrm{~mm}$ no mês de janeiro e os valores mínimos são de $28 \mathrm{~mm}$ com ocorrência em junho.

\section{Herbicidas escolhidos para o estudo}

Foram escolhidos para o estudo os herbicidas clomazone, quinclorac, bentazone, 2,4-D e propanil, que são os mais empregados na cultura de arroz irrigado na região central do estado, conforme pesquisa feita nas casas agrícolas da região e informações do Departamento de Fitotecnia da UFSM.

O fator determinante para a escolha desses herbicidas foi o emprego em grande escala no estado do RS, principalmente na região de Santa Maria, onde há grande produção de arroz, além da falta de informações científicas relacionadas com a poluição das águas por herbicidas nessa região.

$\mathrm{Na}$ Tabela 1 estão relacionadas algumas propriedades dos herbicidas escolhidos para o estudo ${ }^{13-15}$.

\section{Critérios adotados para avaliação}

As propriedades físico-químicas dos herbicidas usados nos critérios de avaliação do potencial de risco para ambientes aquáticos foram a constante da lei de Henry $\left(\mathrm{K}_{\mathrm{H}}\right)$, solubilidade em água, coeficiente de partição octanol-água $\left(\mathrm{K}_{\mathrm{ow}}\right)$, coeficiente de adsorção à matéria orgânica do solo $\left(\mathrm{K}_{\mathrm{oc}}\right)$, constante de ionização ácida $(\mathrm{pKa})$ ou básica $(\mathrm{pKb})$, tempo de meia-vida $\left(\mathrm{t}_{1 / 2}\right)$, no solo e na água ${ }^{13,15}$. Segundo Barceló e Hennion ${ }^{13}$, pesticidas ácidos são os que possuem $\mathrm{pKa}<3-4$, básicos $\mathrm{pKa}>10$; polares tem valores de $\log \mathrm{K}_{\text {ow }}$ abaixo de 1,5; não polares tem valores de $\log \mathrm{K}_{\text {ow }}$ acima de 4 , entre os dois valores são considerados moderadamente polares e, os com $\log \mathrm{K}_{\mathrm{ow}}>3,0$ sofrem bioacumulação. A maioria dos valores do índice de GUS foram calculados por não terem sido encontrados na literatura. Para o cálculo foram utilizados os dados da Tabela 2 e a seguinte equação: GUS $=\log \left(\mathrm{t}_{1 / 2}\right.$ solo $) \times\left(4-\log \mathrm{K}_{\mathrm{OC}}\right) \mathrm{O}$ índice de GUS avalia o potencial de determinado composto ser lixiviado,

Tabela 1. Informações dos herbicidas usados no estudo

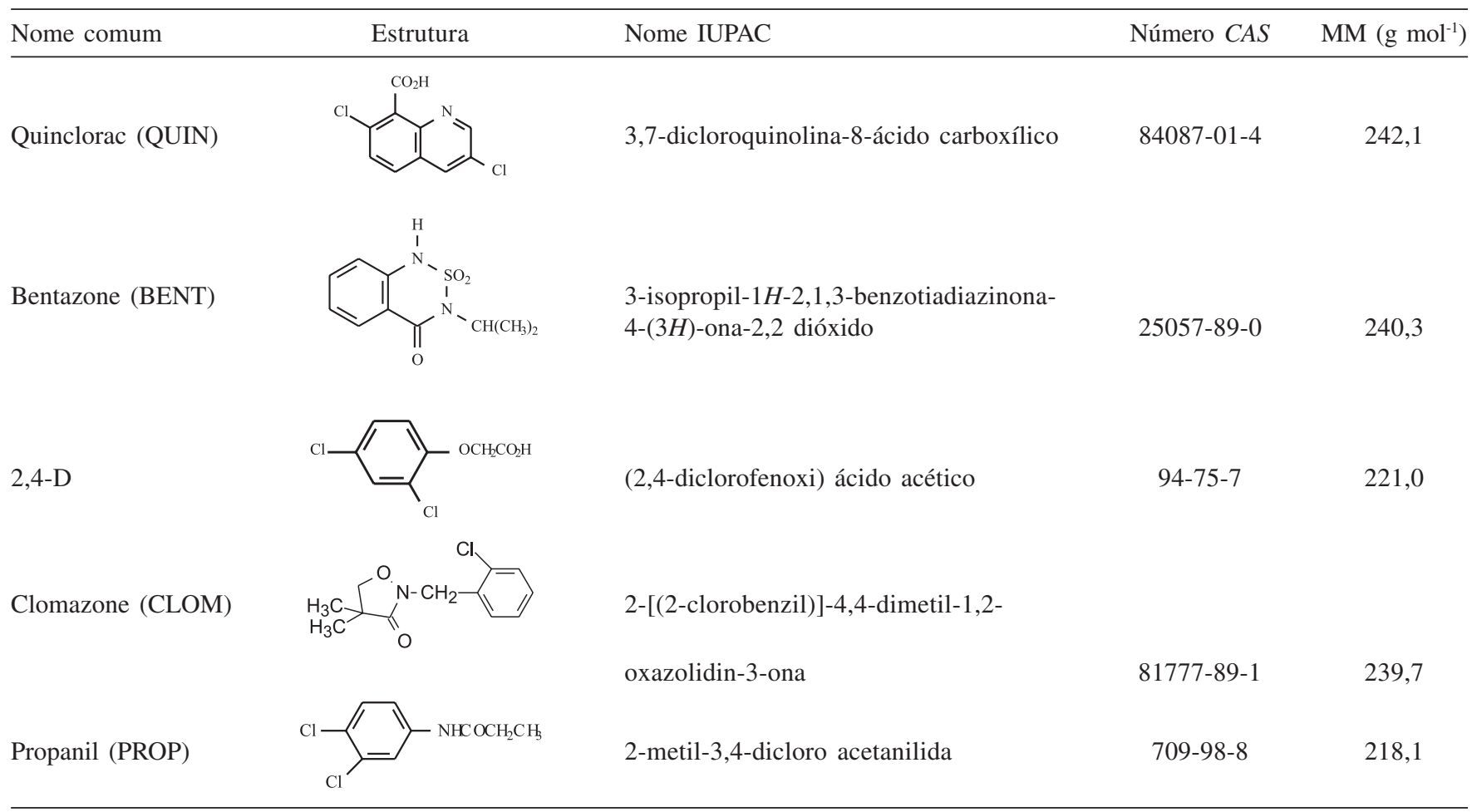

$\mathrm{MM}=$ massa molecular 
atingindo águas subterrâneas e seu valor serve como ferramenta auxiliar para identificação de pesticidas a serem priorizados nas atividades de monitoramento ambiental in $l o c o^{16}$.

O comportamento dos pesticidas no ambiente deve ser diferente entre condições temperadas e tropicais. Segundo Castillo et al. ${ }^{17}$, alguns dados sugerem que as taxas de degradação devem ser mais altas em países tropicais, devido à temperatura mais elevada e à radiação mais intensa.

\section{Critérios usados para avaliar o potencial de lixiviação}

Para avaliar o risco potencial de poluição de águas subterrâneas da região pelos herbicidas em estudo, foram usados os seguintes critérios de "screening": Critérios da EPA e índice GUS ${ }^{13,15}$.

Para avaliar a possibilidade dos herbicidas atingirem as águas superficiais foram utilizados os critérios de "screening" sugeridos pelo método de Goss ${ }^{15}$.

\section{Critérios da EPA}

Em resumo, os critérios de "screening" sugeridos pela EPA na análise preliminar de riscos de poluição de águas subterrâneas por pesticidas são os seguintes: solubilidade em água $>30 \mathrm{mg} \mathrm{L}^{-1}$; coeficiente de adsorção à matéria orgânica do solo $\left(\mathrm{K}_{\mathrm{oc}}\right)<300-500$; constante da Lei de Henry $\left(\mathrm{K}_{\mathrm{H}}\right)<10^{-2} \mathrm{~Pa} \mathrm{~m}^{3} \mathrm{~mol}^{-1}$; especiação: negativamente carregado a $\mathrm{pH}$ normal do ambiente ( $\mathrm{pH} 5-8)$; meiavida no solo > 14-21 d; meia-vida na água > 175 d; condições de campo que favorecem a percolação no solo: pluviosidade anual > $250 \mathrm{~mm}$; aqüífero não confinado e solo poroso.

\section{Índice GUS de vulnerabilidade de águas subterrâneas}

O índice GUS é calculado através dos valores de meia-vida do composto no solo e do coeficiente de adsorção à matéria orgânica do solo ([GUS $=\left(\log t_{1 / 2}\right.$ no solo). $\left.\left.\left(4-\log \mathrm{K}_{\mathrm{oc}}\right)\right]\right)$, não levando em consideração outras propriedades, como por ex., solubilidade em água. As faixas de classificação dos compostos de acordo com sua tendência à lixiviação são GUS $<1,8$ - não sofre lixiviação; $1,8<$ GUS $<$ 2,8 - faixa de transição e GUS > 2,8 - provável lixiviação.

\section{Método de Goss}

Os critérios propostos por esse método para a avaliação do potencial de poluição de águas superficiais por pesticidas, são: - alto potencial de transporte associado ao sedimento (APTAS): meia-vida no solo $\geq 40 \mathrm{~d}$ e $\mathrm{K}_{\mathrm{oc}}=1000$ ou meia-vida no solo $\geq 40 \mathrm{~d}$ e $\mathrm{K}_{\mathrm{oc}} \geq 500$ e solubilidade em água $\leq 0,5 \mathrm{mg} \mathrm{L}^{-1}$;

- baixo potencial de transporte associado ao sedimento (BPTAS): meia-vida no solo $<1 \mathrm{~d}$ ou meia-vida no solo $\leq 40 \mathrm{~d}, \mathrm{~K}_{\mathrm{oc}} \leq 500$ e solubilidade em água $\geq 0,5 \mathrm{mg} \mathrm{L}^{-1}$ ou meia-vida no solo $\leq 2 \mathrm{~d} \mathrm{e} \mathrm{K_{oc }}$

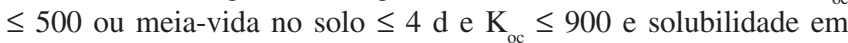

água $\geq 0,5 \mathrm{mg} \mathrm{L}^{-1}$ ou meia-vida no solo $\leq 40 \mathrm{~d} \mathrm{e}_{\mathrm{oc}} \leq 900$ e solubilidade em água $\geq 2 \mathrm{mg} \mathrm{L}^{-1}$;

- alto potencial de transporte dissolvido em água (APTDA): meiavida no solo $>35 \mathrm{~d}, \mathrm{~K}_{\mathrm{oc}}<1.000 .000$ e solubilidade em água $\geq 1 \mathrm{mg}$ $\mathrm{L}^{-1}$ ou $\mathrm{K}_{\mathrm{oc}} \leq 700$ e solubilidade em água entre 10 e $100 \mathrm{mg} \mathrm{L}^{-1}$; - maixo potencial de transporte dissolvido em água (BPTDA): $\mathrm{K}_{\mathrm{oc}}$ $\geq 1.000 .000$ ou meia-vida no solo $\leq 1 \mathrm{~d} \mathrm{e} \mathrm{K}_{\text {oc }} \leq 100$ ou meia-vida no solo $<35 \mathrm{~d}$ e solubilidade em água $<0,5 \mathrm{mg} \mathrm{L}^{-1} \mathrm{e}$

- as substâncias que não se enquadram em nenhum dos critérios acima são consideradas como tendo potencial médio para poluírem águas superficiais.

A Tabela 2 relaciona as propriedades físico-químicas dos pesticidas que são utilizadas para avaliar seu potenciail de poluição para as águas ${ }^{13-15}$.

Pode-se verificar que na Tabela 2 as propriedades físico-químicas entre os cinco herbicidas estudados são bem diferentes entre si, dessa forma não se podem fazer generalizações sobre os destinos e impactos desses herbicidas no ambiente. Entretanto, é possível avaliar teoricamente, através das propriedades físico-químicas dos herbicidas, se apresentam algum risco potencial de poluição das águas. Para isso, emprega-se, geralmente, alguns critérios de "screening", como por ex. os adotados pela EPA, o índice de GUS e o método de Goss ${ }^{15}$.

\section{Amostragem}

Após realizada a avaliação teórica preliminar foram coletadas amostras de água de rios na região produtora de arroz nos afluentes dos Rios Vacacaí-Mirim (80 amostras) e Vacacaí (60 amostras), nas safras 2001/02 e 2002/03, para poder se comparar os dados obtidos pelo método de Goss na avaliação teórica. $\mathrm{O}$ intervalo entre as coletas foi, em ambas as safras, quinzenal para a Bacia do Vacacaí e semanal para a Bacia do Vacacaí-Mirim, sempre na época de maior atividade de aplicação dos herbicidas. Efetuou-se sempre uma coleta anterior a esse período e uma posterior.

A área estudada pertence à Bacia Hidrográfica dos Rios Vacacaí e Vacacaí-Mirim que está localizada na Depressão Central do Rio Grande do Sul. A área limita ao leste no município de Cachoeira do Sul, distante aproximadamente $180 \mathrm{~km}$ de Porto Alegre pela BR-290, ao norte por Santa Maria e Restinga Seca; ao oeste por São Gabriel e ao sul por Caçapava do Sul. Nesta área de abrangência, optou-se pela amostragem em seis locais da Bacia do Rio Vacacaí: Passo do Verde; Passo da Lagoa; Passo do Rocha; Rio São Sepé; Rio Santa Bárbara e Restinga Seca.

Para a Bacia do Rio Vacacaí-Mirim, uma extensão de $40 \mathrm{~km}$ foi avaliada, desde o Distrito de Arroio Grande, no município de Santa Maria, até o município de Restinga Seca, onde predomina o cultivo de arroz irrigado em lavouras de pequeno porte. Nesta Bacia, foram selecionados cinco pontos para amostragem. Foi estabelecido um ponto denominado "branco", por supostamente não receber contribuição de águas de lavoura de arroz irrigado. Este

Tabela 2. Propriedades físico-químicas dos herbicidas que permitem avaliar o potencial de risco para ambientes aquáticos

\begin{tabular}{|c|c|c|c|c|c|c|c|c|}
\hline Herbicida & $\begin{array}{c}\text { Solubilidade } \\
\text { em água }\left(\mathrm{mg} \mathrm{L}^{-1}\right)\end{array}$ & $\begin{array}{c}\mathrm{K}_{\mathrm{oc}} \\
\left(\mathrm{cm}^{3} \mathrm{~g}^{-1}\right)\end{array}$ & $\log K_{\text {ow }}$ & $\begin{array}{c}\mathrm{PV} \\
(\mathrm{mPa})\left(20{ }^{\circ} \mathrm{C}\right)\end{array}$ & $\mathrm{pKa}$ & $\begin{array}{c}\mathrm{K}_{\mathrm{H}} \\
\left(\mathrm{Pa} \mathrm{m}^{3} \mathrm{~mol}^{-1}\right)\end{array}$ & $\mathrm{t}_{1 / 2}$ & água $(\mathrm{d})$ \\
\hline Quinclorac & $0,065\left(20^{\circ} \mathrm{C}\right)$ & 36 & $-1,15 \mathrm{pH} 7,0$ & $<0,01$ & 4,34 & $<3,72 \cdot 10^{-2}$ & & \\
\hline Bentazone & $570\left(20^{\circ} \mathrm{C}\right)$ & 34 & 5,8 pH 5,0 & 0,17 & $3,2-3,3$ & $7,4.10^{-5}$ & 14 & \\
\hline 2,4-D & $311\left(25^{\circ} \mathrm{C}\right)$ & 60 & $2,6-2,8$ & $1,86 \cdot 10^{-2}$ & 2,73 & $1,32 \cdot 10^{-5}$ & $<7$ & 7,5 \\
\hline Clomazone & $1100\left(25^{\circ} \mathrm{C}\right)$ & $150-562$ & 2,54 & 19,2 & & $4,19 \cdot 10^{-3}$ & $30-135$ & $>30$ \\
\hline Propanil & $130\left(25^{\circ} \mathrm{C}\right)$ & $239-800$ & 3,3 & 0,026 & & $3,6.10^{-3}$ & 2 & $1-2$ \\
\hline
\end{tabular}

$\mathrm{d}=$ dias, $\mathrm{PV}=$ pressão de vapor 
ponto localiza-se próximo à nascente do Rio Três Barras.

A amostragem da água nos rios foi realizada em três pontos: uma no centro do leito e as demais nas proximidades das margens direita e esquerda, utilizando-se uma garrafa com capacidade para $2 \mathrm{~L}$ contendo orifícios da metade da garrafa até a extremidade superior. A garrafa coletora foi acoplada a um suporte com peso, permitindo coletar água desde a superfície até o fundo do rio. As amostras de água de lavoura foram coletadas diretamente em frasco de vidro âmbar, $10 \mathrm{~cm}$ abaixo da superfície da lâmina de água. Após coletadas, as amostras foram armazenadas em frasco de vidro âmbar, acidificadas com $\mathrm{H}_{3} \mathrm{PO}_{4}$ 1:1 (v/v) e sob refrigeração transportadas até o laboratório para serem analisadas.

\section{Descrição do procedimento de análise}

Todas as amostras foram analisadas conforme descrito por Primel $^{18}$ e Zanella et al. ${ }^{19}$. Alíquotas de $250 \mathrm{~mL}$ de amostra foram acidificadas e pré-concentradas em cartuchos contendo $200 \mathrm{mg}$ de C18. A eluição foi efetuada com 2 vezes $500 \mu \mathrm{L}$ de metanol. Uma alíquota de $20 \mu \mathrm{L}$ foi injetada no sistema cromatográfico contendo uma coluna Bondesil C18 $(250 \times 4,6 \mathrm{~mm}$ i.d; $5 \mu \mathrm{m})$ com pré-coluna do mesmo material. A eluição foi efetuada com mistura metanol e água (60:40, v/v), ajustada a pH 4,0 com ácido fosfórico, utilizan-

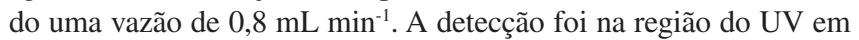
$220 \mathrm{~nm}$.

\section{RESULTADO E DISCUSSÃO}

\section{Águas de superfície}

De acordo com os critérios de Goss usados para avaliar se um pesticida ao ser usado na agricultura pode atingir águas de superfície, pode-se dividí-los entre aqueles que podem ser transportados dissolvidos em água e aqueles que são transportados associados ao sedimento em suspensão. Assim, dos herbicidas em estudo o clomazone e o propanil indicam um alto potencial de poluição de águas de superfície (APTDA) porque podem ser transportados dissolvidos em água. O quinclorac apresenta um potencial médio (MPTA) com relação a esse parâmetro, enquanto o bentazone e o 2,4-D indicam um baixo potencial de poluição (BPTDA) de água de superfície no parâmetro solubilidade em água. Quanto ao transporte no sedimento em suspensão, os herbicidas clomazone, 2,4-D e propanil indicam baixo potencial de poluição de águas de superfície (BPTAS), e o bentazone e quinclorac indicam um potencial médio (MPTAS) de poluição dessas águas.

\section{Águas subterrâneas}

A Tabela 3 apresenta os resultados da análise do potencial de poluição de águas subterrâneas usando os critérios de "screening" propostos pela EPA. Foram considerados as propriedades apresen- tadas na Tabela 2 e os critérios citados anteriormente para analisar o risco dos pesticidas usados na região atingirem águas subterrâneas.

Apesar da falta de alguns dados para esta análise, não disponíveis na literatura, pode-se classificar alguns dos herbicidas como compostos que apresentam maior probabilidade de atingir as águas subterrâneas, pois apresentam considerável solubilidade em água, baixa adsorção à matéria orgânica do solo e tempo de meia-vida no solo relativamente alto.

Foram considerados poluentes em potencial somente aqueles princípios ativos para os quais a maioria das propriedades físicoquímicas disponíveis indicava uma possibilidade de poluição das águas subterrâneas. Quando as informações disponíveis foram insuficientes para se concluir sobre o potencial de poluição do herbicida, o resultado foi apresentado como inconclusivo.

Segundo Dores et al. ${ }^{15}$ compostos classificados na faixa de transição e de provável lixiviação de acordo com o índice de GUS requerem investigação adicional, usando-se procedimentos mais detalhados. Compostos classificados como improváveis de sofrerem lixiviação podem, seguramente, ser considerados como não poluentes de águas subterrâneas.

Considerando esta afirmação e os critérios da EPA pode-se dizer que para os herbicidas bentazone, 2,4-D, clomazone e propanil seriam recomendados estudos complementares sobre a possibilidade de poluição de águas subterrâneas na região. Com relação ao quinclorac, por falta de dados sobre diversas de suas propriedades, nada se pode afirmar sobre seu potencial de poluição.

Utilizando-se o índice de GUS, bentazone e clomazone podem ser considerados contaminantes em potencial, propanil como não contaminante, 2,4-D de transição e quinclorac inconclusivo.

Considerando a caracterização físico-hídrica do perfil do solo da área, destaca-se uma zona de maior macroporosidade nas camadas de 30 a $45 \mathrm{~cm}$ e de 45 a $60 \mathrm{~cm}$ de profundidade, com maiores valores relativos de condutividade hidráulica do perfil quando comparadas com as camadas superficiais. As camadas intermediárias contrastam com a camada arável, que se apresenta com microporosidade mais elevada. As condições texturais do perfil explicam esta modificação, por apresentarem elevados teores de silte nas camadas superficiais $(64 \%)$ em relação às intermediárias (55 e $38 \%$ ), contribuindo para os baixos valores de condutividade hidráulica saturada ${ }^{20}$. A possibilidade de lixiviação reduz-se bastante pelo fato deste solo ser mais arenoso na camada de 30 a $60 \mathrm{~cm}$, e ter mais silte e argila na camada superficial. Assim, o movimento da água no perfil é menor na superfície do solo e é por isto que retém a água.

Embora aborde apenas algumas propriedades dos herbicidas sem levar em consideração as particularidades de solo e clima, a análise do potencial de lixiviação, segundo os critérios propostos, pode ser uma ferramenta para a avaliação inicial do potencial de poluição ambiental por herbicidas, pois a conjunção de altas doses, alto potencial de lixiviação e solos com baixa capacidade de

Tabela 3. Resultados da avaliação de risco de poluição das águas subterrâneas com base nos critérios estabelecidos pela EPA

\begin{tabular}{lcccccc}
\hline Herbicidas & Solubil. & $\mathrm{K}_{\mathrm{oc}}$ & $\mathrm{t}_{1 / 2}$ solo & $\mathrm{t}_{1 / 2}$ água & $\mathrm{K}$ & $\mathrm{K}_{\mathrm{H}}$ \\
\hline QUIN & $\mathrm{N}$ & $\mathrm{A}$ & & $\mathrm{N}$ & $\mathrm{A}$ & $\mathrm{I}$ \\
BENT & $\mathrm{A}$ & $\mathrm{A}$ & $\mathrm{A}$ & $\mathrm{N}$ & $\mathrm{A}$ & $\mathrm{A}$ \\
$2,4-\mathrm{D}$ & $\mathrm{A}$ & $\mathrm{N}$ & $\mathrm{N}$ & $\mathrm{A}$ & $\mathrm{P}$ \\
CLOM & $\mathrm{A}$ & $\mathrm{A}$ & $\mathrm{A}$ & $\mathrm{N}$ & $\mathrm{A}$ \\
PROP & $\mathrm{A}$ & $\mathrm{A}$ & $\mathrm{N}$ & $\mathrm{A}$ & PC \\
\hline
\end{tabular}

$\mathrm{N}$ = não atende ao critério; $\mathrm{A}=$ atende ao critério como potencialmente perigoso; $\mathrm{I}=$ inconclusivo; $\mathrm{PC}=$ contaminante em potencial; $\mathrm{NC}$ = não contaminante; em branco $=$ dado não disponível; Solubil. $=$ Solubilidade em água 
retenção sugerem uma situação de alto risco do herbicida para o meio ambiente.

\section{Análise de amostra de água de superfície da região em estudo}

Pode-se verificar nas Tabelas 4 e 5, onde são demonstrados os resultados obtios para os Rios Vacacaí-Mirim e Vacacaí, respectivamente, que clomazone e propanil são os herbicidas que apresentaram maior percentual de amostras positivas. Isso pode ser devido à grande quantidade de clomazone aplicado em lavouras da região e à alta concentração de propanil que é aplicada na lavoura, como pode ser observado na Tabela 6, que apresenta os valores de resíduos de herbicidas obtidos para 63 amostras de águas coletadas em lavouras na região na safra de 2000-2001. Os resultados indicam que clomazone e propanil são os herbicidas mais freqüentemente detectados na água de lavoura, evidenciando que provavelmente eles sejam os mais utilizados em lavouras da região. Esses resultados confirmam a análise preliminar, onde se utilizou o método de Goss, no qual clomazone e propanil foram classificados como tendo APTDA.

Tabela 4. Número de amostras positivas obtidas para 80 amostras de água do Rio Vacacaí-Mirim e afluentes, coletadas nos anos de 2001-2003

\begin{tabular}{lccccc}
\hline Herbicidas & < LOQ & $\begin{array}{c}0,1-0,5 \\
\mu \mathrm{g} \mathrm{L}^{-1}\end{array}$ & $\begin{array}{c}0,5-2,0 \\
\mu \mathrm{g} \mathrm{L}^{-1}\end{array}$ & $\begin{array}{c}>2,0 \\
\mu \mathrm{g} \mathrm{L}^{-1}\end{array}$ & $\%$ Pos $^{1}$ \\
\hline QUIN & 76 & 0 & 1 & 3 & 5 \\
BENT & 70 & 0 & 6 & 4 & 12,5 \\
2,4-D & 75 & 0 & 9 & 1 & 12,5 \\
CLOM & 68 & 0 & 6 & 6 & 15 \\
PROP & 68 & 0 & 9 & 3 & 15 \\
\hline
\end{tabular}

${ }^{1}$ percentagem de amostras positivas.

Tabela 5. Número de amostras positivas obtidas para 60 amostras de água do Rio Vacacaí e afluentes, coletadas nos anos de 20012003

\begin{tabular}{lccccc}
\hline Herbicidas & $<\mathrm{LOQ}$ & $\begin{array}{c}0,1-0,5 \\
\mu \mathrm{g} \mathrm{L}^{-1}\end{array}$ & $\begin{array}{c}0,5-2,0 \\
\mu \mathrm{g} \mathrm{L}^{-1}\end{array}$ & $\begin{array}{c}>2,0 \\
\mu \mathrm{g} \mathrm{L}^{-1}\end{array}$ & $\% \operatorname{Pos}^{1}$ \\
\hline QUIN & 0 & 0 & 0 & 0 & 0 \\
BENT & 54 & 0 & 0 & 6 & 10 \\
2,4-D & 44 & 0 & 2 & 4 & 10 \\
CLOM & 44 & 0 & 4 & 12 & 27 \\
PROP & 49 & 0 & 7 & 4 & 18 \\
\hline
\end{tabular}

${ }^{1}$ percentagem de amostras positivas.

Tabela 6. Número de amostras positivas obtidas para as 63 amostras de água coletadas em lavouras na safra 2000-2001.

\begin{tabular}{lccccc}
\hline Herbicidas & $<\mathrm{LOQ}$ & $\begin{array}{c}0,1-0,5 \\
\mu \mathrm{g} \mathrm{L}^{-1}\end{array}$ & $\begin{array}{c}0,5-2,0 \\
\mu \mathrm{g} \mathrm{L}^{-1}\end{array}$ & $\begin{array}{c}>2,0 \\
\mu \mathrm{g} \mathrm{L}^{-1}\end{array}$ & $\% \operatorname{Pos}^{1}$ \\
\hline QUIN & 60 & 0 & 2 & 1 & 5 \\
BENT & 60 & 0 & 3 & 0 & 5 \\
2,4-D & 54 & 0 & 4 & 5 & 14 \\
CLOM & 42 & 0 & 5 & 16 & 33 \\
PROP & 52 & 0 & 5 & 6 & 17 \\
\hline
\end{tabular}

\footnotetext{
${ }_{1}^{1}$ percentagem de amostras positivas.
}

\section{CONCLUSÃO}

Esse trabalho demonstra que a quantidade de herbicidas usados nas lavouras de arroz irrigado influenciam diretamente os níveis de herbicidas que ocorrem nas águas de superfície das proximidades. Para reduzir a quantidade de herbicidas que atingem as águas de superfície são necessários programas de gerenciamento e conscientização para minimizar a quantidade aplicada.

Herbicidas usados na cultura do arroz irrigado têm um efeito prejudicial potencial para a vida aquática, pois a drenagem da água da lavoura de arroz irrigado coincide com a época de reprodução dos peixes. Então, todo sistema de cultivo de arroz que libera água para o meio ambiente precisa ser monitorado com relação à concentração de herbicidas, e planos de gerenciamento, de manejo da cultura e de desempenho para proteger a vida aquática precisam ser implementados.

Considerando a grande importância de se conhecer o nível de herbicidas nas águas, mais estudos são necessários para determinar exatamente os processos de dispersão de herbicidas aplicados na lavoura, tais como volatilização, degradação por microrganismos e luz solar, e adsorção no solo, pois os herbicidas podem ser prejudiciais à saúde humana e ao meio ambiente, demonstrando esses efeitos mesmo em pequenas concentrações.

\section{AGRADECIMENTOS}

Ao CNPq pela bolsa de Doutorado concedida à E.G. Primel.

\section{REFERÊNCIAS}

1. Colborn, T.; Dumanoski, D.; Myers, J. P.; O Futuro Roubado, L \& PM Ed.: Porto Alegre, 1997.

2. Bizuik, M.; Przyjazny, A.; Czerwinski, J.; Wiergowski, M.; J. Chromatogr., A 1996, 754, 103.

3. Baird, C.; Química Ambiental, Ed. Bookman: Porto Alegre, 2 ${ }^{\mathrm{a}}$ ed., 2002.

4. Chomenko, L.; Epagri 1997, 97, 66.

5. Hermes, L. C.; Epagri 1997, 97, 68.

6. Traub-Eberhard, U.; Pest. Sci. 1995, 43, 121.

7. Logan, T. J.; Soil \& Tillage Research 1994, 30, 75.

8. Lanças, F. M.; Dórea, H. S.; Barbirato, M. A.; Pesticidas R. Ecotoxicol. e Meio Ambiente 1997, 7, 109.

9. http://www.sindag.com.br/db/arqs/graficokgha.xls, acessada em Abril 2004.

10. Martins, D, I.; Mídio, A. F.; Revista Brasileira de Toxicologia 1998, 11, 13.

11. AGRIANUAL; Anuário da Agricultura Brasileira, FNP Consultoria \& Comércio: Ed. Argos, 2000.

12. Instituto Riograndense do Arroz (IRGA); Lavoura Arrozeira Gaúcha: Perfil do Sistema Produtivo Porto Alegre-RS, 1996.

13. Barceló, D.; Hennion, M. C. Em Water-Techniques and Instrumentation in Analytical Chemistry; $1^{\text {st }}$ ed., Elsevier Science B. V: Amsterdam, 1997, cap. 19.

14. Tomlin, C.D.S.; The e-Pesticide Manual, $13^{\text {th }}$ ed., Versão 3.0, Londres, 2003-2004.

15. Dores, E. F. G. C.; De-Lamonica-Freire, E. M.; Quim. Nova 2001, 24, 27.

16. Ferracini, V. L.; Pessoa, M. C. V. P.; Silva, A. S.; Spadotto, C. A.; Pesticidas: R. Ecotoxicol. e Meio Ambiente 2001, 11, 1.

17. Castillo, L.E.; De La Cruz, E.; Ruepert, C.; Environ. Toxicol. Chem. 1997, 16, 41.

18. Primel, E. G.; Tese de Doutorado, Universidade Federal de Santa Maria, Brasil, 2003.

19. Zanella, R.; Primel, E. G.; Gonçalves, F. F.; Kurz, M. H. S.; Mistura, C. M.; J. Sep. Sci. 2003, 26, 935.

20. Righes, A. A.; Resumos do Simpósio sobre Alternativas ao Manejo Tradicional de utilização das Várzeas do RS, Porto Alegre, Brasil, 1984. 